\title{
Los Pirineos, entre las políticas europeas y las iniciativas locales de cooperación transfronteriza: el caso de la Cerdaña
}

\author{
Matteo Berzi \\ Universitat Autònoma de Barcelona. Departament de Geografia \\ matteo.berzi@uab.cat
}

\section{Margarita Castañer i Vivas}

Universitat de Girona. Departament de Geografia

mita.castaner@udg.edu

\section{Resumen}

Este artículo tiene como objetivo analizar el papel de la cooperación transfronteriza local en las áreas de fronteras periféricas. Específicamente, nos preguntamos si las sinergias entre las políticas comunitarias de cooperación territorial europea (CTE), por un lado, y las iniciativas transfronterizas locales, por otro lado, representan una estrategia territorial de desarrollo endógeno válida en dichas áreas. Los territorios de frontera (borderlands) muy a menudo coinciden con lugares geográficamente remotos (ex. áreas de montaña o zonas rurales o costeras), generalmente lejanos de los principales centros económicos y urbanos de crecimiento. Los Pirineos constituyen, en este sentido, un área de estudio privilegiada. Desde finales de los ochenta, en este espacio transfronterizo se han generado muchos proyectos transfronterizos y cuenta, a la vez, con diferentes organismos de cooperación regional y local. El análisis del caso de estudio de la Cerdaña proporciona los datos empíricos que fundamentan la hipótesis que la cooperación transfronteriza local contribuye a la integración de los territorios fronterizos periféricos. El estudio demuestra como las relaciones históricas de vecindad, la fuerte identidad local y el hecho de compartir necesidades comunes, son factores fundamentales para el desarrollo de una cooperación transfronteriza local exitosa, estable, multitemática y de largo plazo.

Palabras clave: Cerdaña; procesos bottom-up; enfoque top-down; cooperación transfronteriza; desarrollo local endógeno 
Resum. Els Pirineus, entre les politiques europees $i$ les iniciatives locals de cooperació transfronterera: el cas de la Cerdanya

Aquest article té com a objectiu analitzar el paper de la cooperació transfronterera local a les àrees de fronteres periferiques. Específicament, ens preguntem si les sinergies entre les polítiques comunitàries de cooperació territorial europea (CTE), d'una banda, i les iniciatives transfrontereres locals, d'una altra, representen una estratègia territorial de desenvolupament endogen vàlida en aquestes àrees. Els territoris de frontera (borderlands) molt sovint coincideixen amb llocs geogràficament remots (ex. àrees de muntanya o zones rurals o costaneres), generalment allunyats dels principals centres econòmics i urbans de creixement. Els Pirineus constitueixen, en aquest sentit, una àrea d'estudi privilegiat. Des de finals dels vuitanta, en aquest espai transfronterer s'han generat molts projectes i compta, alhora, amb diferents organismes de cooperació regional i local. L'anàlisi del cas d'estudi de la Cerdanya proporciona les dades empíriques de suport de la nostra hipòtesi que la cooperació transfronterera local contribueix a la integració dels territoris fronterers periferics. L'estudi demostra com les relacions històriques de veïnatge, la forta identitat local i el fet de compartir necessitats comunes són factors fonamentals per al desenvolupament d'una cooperació transfronterera local d'èxit, estable, multitemàtica i de llarg termini.

Paraules clau: Cerdanya; processos bottom-up; enfocament top-down; cooperació transfronterera; desenvolupament local endogen

Résumé. Les Pyrénées entre les politiques européennes et les initiatives locales de coopération transfrontalière: le cas de la Cerdagne

L'article vise à analyser le rôle de la coopération transfrontalière locale dans les domaines des frontières périphériques. Plus précisément, nous nous demandons si les synergies entre les politiques européennes de coopération territoriale européenne (CTE), d'une part, et les initiatives transfrontalières locales, d'autre part, représentent une stratégie territoriale valable de développement endogène de ces domaines. Les territoires frontaliers (les borderlands) coïncident très souvent avec des sites géographiquement distants (ex. les zones de montagne ou les zones rurales ou côtières), généralement éloignées des grands centres de croissance économique et urbaine. Les Pyrénées sont, dans ce sens, une zone d'étude privilégiée. Depuis la fin des années quatre-vingt dans cet espace de la frontière de nombreux projets transfrontaliers ont été générés et, en même temps, à partir de différents organismes de coopération régionale et locale. L'analyse de l'étude de cas de la Cerdagne fournit des données empiriques qui soutiennent notre hypothèse selon laquelle la coopération transfrontalière locale conduit à l'intégration des territoires frontaliers marginaux. L'étude montre comment les relations de voisinage historique, une forte identité locale et le partage des besoins communs, sont fondamentaux pour le développement d'un des facteurs de coopération transfrontalièreréussie, stable, multi-thématique et de longue durée.

Mots-clés: Cerdagne; processus bottom-up; approche top-down; coopération transfrontalière; développement local endogène

\section{Abstract. The Pyrenees between European policies and local initiatives for cross-border cooperation: The case of Cerdanya}

The article analyzes the role of local cross-border cooperation in peripheral border areas. Specifically, we inquire as to whether the synergies between European Territorial Cooperation (ETC) policy on the one hand, and local cross-border initiatives on the other, represent a valid territorial strategy for endogenous development in these areas. Border- 
lands frequently coincide with geographically remote locations (i.e., mountain or rural or coastal areas) that are often far from major economic and urban centers of growth. In this regard, the Pyrenees constitute a privileged area of study. Since the end of the 1980s, many cross-border projects have been implemented in this transboundary space and different regional and local cooperation agencies have been set up. A case study of the Cerdanya provides empirical data to support our hypothesis that local cross-border cooperation is leading to the integration of peripheral borderlands. The study demonstrates how historical neighbourhood relationships, a strong local identity and sharing common needs are key factors in the development of successful, stable, multi-thematic and local cross-border cooperation in the long term.

Keywords: Cerdanya; bottom-up processes; top-down approach; cross-border cooperation; local endogenous development

\author{
Sumario \\ 1. Introducción 4. Caso de estudio de la Cerdaña \\ (análisis y resultados) \\ 2. La cooperación transfronteriza \\ top-down y bottom-up 5. Conclusiones \\ 3. Metodología Referencias bibliográficas
}

\title{
1. Introducción
}

En Europa, en las últimas décadas, los cambios inducidos en la geopolítica mundial (fin de la Guerra Fría y afirmación de la globalización) y en la escala regional (el proceso de integración europea) han situado las regiones fronterizas (el 37,5\% de la población europea vive en dichas regiones) en el centro de la discusión institucional y académica. En este sentido, recordamos, por un lado, las aportaciones de las instituciones europeas como el Consejo de Europa (Council of Europe, 1980, 1995, 1998) y la Unión Europea (a través de los programas Interreg) para impulsar la cooperación transfronteriza regional y local y, por otro lado, la evolución de los estudios de fronteras (Rumley y Minghi, 1969; Paasi, 1996; Kaplan y Häkli, 2002; Wilson y Donnan, 2012 entre otros).

La literatura académica identifica las áreas alrededor de una frontera (Kaplan y Häkli, 2002) con diferentes denominaciones: entre las más utilizadas encontramos borderlands (Martinez, 1992), cross-border region (Perkmann, 2003), transfrontier regions (Anderson, 1997) o transboundary regions en los análisis realizados desde la perspectiva de las ciencias ambientales (Walmer, 2003; Duffy, 2006). Desde un punto de vista analítico, los estudios de frontera (border studies) se han interesado por definir su delimitación espacial (Schelde y Hoekyeld, 1992); su estructura interna (Martinez, 2002); las dinámicas políticas (Popescu, 2008), socioeconómicas, culturales (Paasi, 1998) y territoriales (Decoville et al., 2013). Van Houtum (2000) ha reconocido en estos estudios tres enfoques principales. Primero, el enfoque de los flujos (flow approach), cuyos exponentes (principal- 
mente ligados a la geografía económica) se interesan por las relaciones económicas entre países o regiones y asumen las fronteras como barrera o limitaciones a dichos flujos. Segundo, el enfoque denominado 'Cooperación Transfronteriza' (cross-border cooperation approach), que, desde los años noventa, agrupa los estudios de las relaciones institucionales entre las entidades fronterizas (regiones y entidades locales) e interpreta la frontera como puente hacia la integración territorial (Perkmann, 2003; Sohn, 2014). Tercero, el llamado 'enfoque de las personas' (people approach), que identifica la frontera como parte de la identidad de los individuos y estudia como dichas 'fronteras' están socialmente y culturalmente reproducidas en el tiempo y en el espacio geográfico (Paasi, 1996).

La región pirenaica (frontera entre España y Francia) ha sido analizada a partir de los tres enfoques. A través del flow approach se han mostrado las limitaciones inducidas por la frontera hispano-francesa para el desarrollo de las infraestructuras viarias (CESEDEN, 1991; Loncán, 2002); el people approach ha sido aplicado para estudiar la percepción y la formación de las identidades locales y nacionales en las poblaciones que viven en áreas de montaña (Sahlins, 1989; Moncusí, 2005, Alland y Alland, 2006). En esta investigación, sin embargo, nos centramos en el enfoque de la cooperación transfronteriza, dado que el artículo tiene como objetivo analizar el papel de la cooperación transfronteriza local (CTF) en las áreas de fronteras periféricas (no urbanas). En relación con este objetivo, nos planteamos la hipótesis que la cooperación transfronteriza local contribuye a la integración de los territorios fronterizos periféricos demostrando como las sinergias entre las políticas comunitarias de cooperación territorial europea (CTE), por un lado, y las iniciativas locales de CTF, por otro lado, puedan generar un desarrollo local endógeno en dichas áreas (Berzi, 2017). Estas regiones sufren a menudo despoblación y el aislamiento económico y social debido a su fragmentación territorial, distancia de los centros de decisión, etc. (European Commission, 2016). Por consiguiente, el artículo pretende enriquecer los estudios de fronteras en la Península Ibérica para aportar nuevas perspectivas analíticas y de estudio de casos que sean comparables con otras áreas periféricas. Por ejemplo, con las investigaciones que se realizan a lo largo de la frontera hispano-portuguesa noroccidental (Paül y Trillo-Santamaría, 2014), meridional (Fernández-Sánchez, 2008) y en la verja de Gibraltar (Haller, 2000).

$\mathrm{El}$ artículo $^{1}$ se estructura en cuatro apartados. En el primero se expone el marco teórico basado en la CTF top-down y bottom-up, con referencia a la

1. Este trabajo ha sido realizado en el marco del doctorado en Geografía de la Universidad Autónoma de Barcelona (UAB) y se enmarca en dos proyecto de investigación. El primero, CSO2013-45257-P, titulado Cooperación territorial en España y la Unión Europea: selección de experiencias de excelencia e innovación para el periodo 2014-20 (COOP-RECOT-II), ha sido subvencionado por el Ministerio de Economía y Competitividad (MINECO) y concedido al equipo RECOT de la UAB (Departamento de Geografía); y el segundo, CSO2011-26151, titulado 25 años de proyectos transfronterizos entre España y Francia en el marco de la UE. Un análisis desde la geopolitica de planes, proyectos, agentes y resultados territoriales, subvencionado por el Ministerio de Ciencia e Innovación (MICINN) y concedido al equipo APTA de la Universidad de Girona. 
frontera hispano-francesa y, además, se discute brevemente sobre el concepto de integración transfronteriza como resultado de las sinergias de ambas dinámicas. Seguidamente, se comenta el enfoque metodológico y se presenta el caso de la Cerdaña, una microrregión geográfica e histórica dividida entre Francia y España desde 1659. En esta sección, se analiza cómo, a pesar de 350 años de división política y administrativa, hay importantes similitudes territoriales de tipo socioeconómico, paisajístico, cultural..., que son la base de las recientes dinámicas de cooperación institucional. En el último apartado se analizan tres proyectos emblemáticos para la CTF local: el nuevo Hospital de la Cerdaña, el futuro matadero compartido y el Plan del Paisaje Transfronterizo. Finalmente se avanzan unas consideraciones en relación con la hipótesis de integración transfronteriza local.

\section{La cooperación transfronteriza top-down y bottom-up}

Se puede definir la cooperación territorial transfronteriza como la «asociación más o menos institucionalizada que se desarrolla entre las autoridades o entidades subestatales de uno o varios estados, cuya máxima expresión es la creación de organismos de cooperación, orientados a la coordinación horizontal y vertical de las políticas y acciones» (Oliveras et al., 2010: 24). Si bien Perkmann analiza la historia de la cooperación trasfronteriza estructurándola en diferentes etapas (2003), en este artículo la analizamos a partir de dos categorías de intervenciones: la primera implementada de arriba hacia abajo (enfoque top-down), fomentada por la Unión Europea y por los estados miembros, y la segunda implementada de abajo hacia arriba (enfoque bottom-up), fomentada por los actores locales y regionales. En este sentido, pensamos que la sinergia entre cooperación bottom-up y top-down representa una estrategia territorial válida para enfrentar los retos de las áreas fronterizas periféricas. Finalmente, proponemos una serie de consideraciones sobre el concepto de integración transfronteriza.

\subsection{Las politicas top-down: efectos en la frontera hispano-francesa}

En esta sección consideramos tres tipologías de iniciativas top-down que, desde los años ochenta, han impulsado la cooperación transfronteriza local y regional en Europa: iniciativas legislativas, introducción de figuras jurídicas y ayuda financiera. Dichas iniciativas, como veremos más adelante, inciden directamente en el caso de estudio.

Desde mediados de los años ochenta, las instituciones europeas impulsan la CTF local y regional a través de importantes iniciativas jurídicas y financieras. En 1980, el Consejo de Europa (CoE), a partir del Convenio Marco de Madrid, inauguró la primera fase "clásica» de la CTF (Perkmann, 2003). La finalidad era incentivar a los países firmantes a promover y facilitar la práctica de la CTF entre las entidades territoriales a lo largo de sus fronteras (regiones y entidades locales), a través del establecimiento de acuerdos internacionales y bilaterales (MHAP, 2014). En este sentido, el primer acuerdo entre España 
y Francia fue el Tratado de Bayona, del 10 de marzo de 1995. Fue un acto fundamental para regular la CTF regional y local a lo largo de la frontera de los Pirineos.

Otra iniciativa importante fue la Carta Europea de Autonomía Local, firmada en 1985 por todos los estados miembros del CoE, donde se establece el derecho de las entidades locales a cooperar con instituciones análogas de otros estados (art.10). Para reforzar el papel de la cooperación transfronteriza, el convenio de Madrid fue ampliado a través de dos protocolos: el Protocolo Adicional en 1995 y el Protocolo en 1998 (Council of Europe, 1995, 1998). Si bien el primero se planteaba reducir los obstáculos a la CTF que surgieron, reafirmando los valores y los derechos de las autoridades locales en la práctica de la cooperación, el segundo extendía la cooperación a los territorios no contiguos (cooperación interterritorial). Los dos actos fueron firmados y ratificados por la mayoría de los gobiernos europeos.

Sin embargo, el primer acto de la Unión Europea para impulsar la CTF local fue la Reforma de los Fondos Estructurales en 1989, con el objetivo de preparar las regiones transfronterizas para el futuro Mercado Único (Harguindéguy, 2007; Allen, 2010). Un instrumento importante de dicha reforma, que constituye aun hoy uno de los factores clave para la aplicación efectiva de la cooperación transfronteriza, fue la iniciativa Interreg. Dicha iniciativa se introdujo con la finalidad de reducir el aislamiento socioeconómico de las regiones fronterizas que sufrían y sufren graves problemas de desarrollo industrial y rural.

En el arco temporal de 25 años se han implementado cuatro programas Interreg para impulsar proyectos de cooperación sobre temáticas diferentes en función del periodo de programación. Los proyectos están principalmente financiados por el Fondo Europeo de Desarrollo Regional (FEDER) ${ }^{2}$. Desde la segunda programación (1994-1999) se definieron diferentes tipologías de cooperación: la cooperación verdaderamente transfronteriza, que tiene como objetivo alcanzar un desarrollo socioeconómico de los espacios fronterizos; la cooperación interregional entre las regiones de la misma área geográfica (ej. Mediterráneo occidental, Espacio Alpino, países bálticos), y la cooperación transnacional, destinada a la generación de redes de entidades territoriales lejanas que comparten los mismos retos y las mismas problemáticas (sobre todo medioambientales).

De las tres líneas, la primera es la que históricamente ha recogido mayor financiación y el mayor número de programas operativos. En la programación actual (2014-2020) se han destinado 6,6 billones de fondos FEDER entre 60 programas de cooperación entre las fronteras internas de la UE. En la tabla 1 se resume la evolución de los programas Interreg a lo largo de la frontera hispano-francesa. Los actores locales y regionales han desarrollado más de 800 proyectos en 27 años. Aunque no dispongamos de los datos generales sobre la

2. En los primeros dos Interreg (Interreg I 1989-1993; Interreg IIA 1994-1999) la financiación procedía también de otros instrumentos financieros de la UE (FEOGA y FSE). 
Tabla 1. Evolución de los programas Interreg de la frontera hispano-francesa

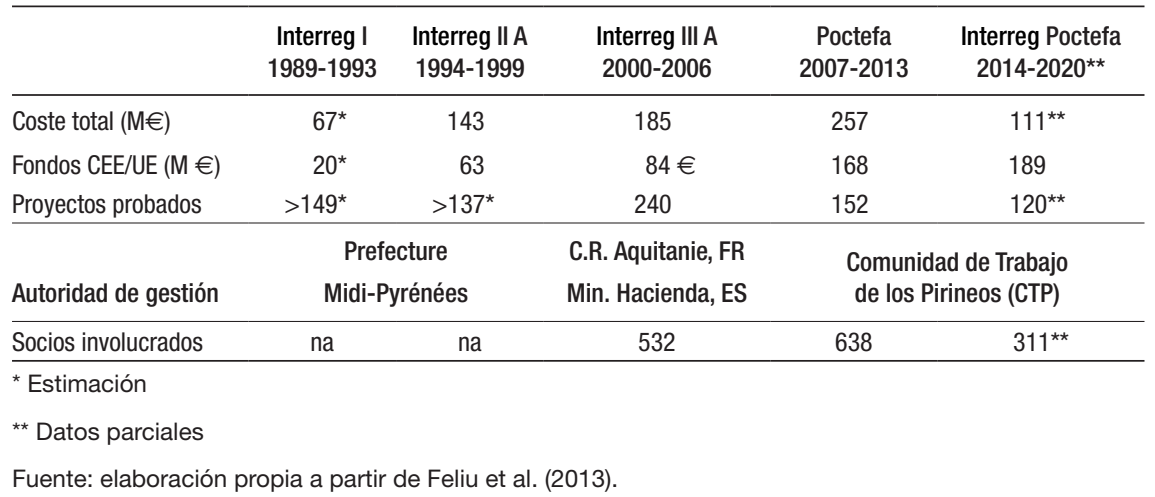

participación de los actores locales en las primeras convocatorias, el número de agentes territoriales involucrados y la aportación financiera ha sido relevante.

La Unión Europea es el principal actor legislativo y financiero para la ayuda de la CTF local y regional. En este sentido, una estrategia importante fue la introducción de figuras jurídicas ad-hoc que facilitan la práctica de la cooperación. Uno de los primeros actos fue la introducción por parte del Consejo de las Comunidades Europeas (CCE) de la Agrupación Europea de Interés Económico (AEIE) en 1985 (CEE n. 2137/85). Dicha agrupación es el primer marco jurídico que permite a las empresas y las instituciones locales «cooperar efectivamente más allá de las fronteras» (Reg. CEE n. 2137/1985). El objetivo final era intentar facilitar a las empresas europeas el acceso al futuro Mercado Único Europeo. A lo largo de la frontera hispano-francesa hay una sola AEIE activa, la Eurociudad Vasca, nacida a principios de los años noventa como resultado de la cooperación entre la Diputación Foral de Guipúzcoa y la Agglomération Côte Basque-Adour (tabla 2) y que hoy cuenta con la participación de cuatros socios más (Consorcio Transfronterizo Bidasoa-Txingudi, la Agglomération Sud Pays Basque, el Ayuntamiento de San Sebastián y la Agencia de Desarrollo Comarcal Oarsoaldea).

Para mejorar la gestión de los proyectos derivados del Interreg en 2006, el legislador comunitario introdujo un instrumento jurídico inédito: la Agrupación Europea de Cooperación Territorial - AECT (CE n. 1082/2006). Las principales causas que impulsaron al legislador a introducir este nuevo instrumento fueron: la ineficacia de los instrumentos existentes hasta el momento (AEIE, consorcios, etc.) y los obstáculos a la cooperación que derivan de las legislaciones nacionales y de las asimetrías institucionales entre agentes locales y regionales (Berzi, 2013). Por eso, la AECT proporciona una personalidad jurídica, enmarcando los límites y su capacidad de acción ${ }^{3}$. A finales de 2017 se habían constituido 68 AECT,

3. El Reglamento reconoce a la AECT, entre otras competencias, la capacidad de «adquirir y enajenar bienes muebles e inmuebles, disponer del personal y soportar en juicio». Los miem- 
incluyendo más de 800 entidades locales, regionales y nacionales de diferentes países miembros. Otras 23 están en curso de constituirse (METIS, 2018). La frontera hispano-francesa cuenta con 7 AECT establecidas a lo largo de toda la frontera pirenaica (tabla 2); es decir, el 10\% del total europeo.

En 2009, el tercer protocolo del Convenio Marco de Madrid introdujo un tercer instrumento legal para promover la CTF, la Agrupación Eurorregional de Cooperación - AEC (Council of Europe, 2009) y, aunque muy pocos estados firmaron el acuerdo y aún menos lo ratificaron, Engl (2007) sostiene que dicho instrumento proporciona muchas competencias a las autoridades locales y regionales. Además, su institucionalización no debería pasar por las autoridades centrales.

\subsection{Las iniciativas bottom-up: efectos en la frontera hispano-francesa}

Unos de los primeros actos de cooperación bottom-up en Europa se llevó a cabo a nivel local a partir de la práctica de "hermanamientos», un movimiento que se inició en la posguerra europea: en 1950, Bapaume (municipio francés) y Moers (municipio alemán) fueron las dos primeras ciudades que avanzaron en esta dirección. Son enlaces simbólicos promovidos por las autoridades locales que tienen como objetivo desarrollar relaciones políticas, sociales y económicas. En 1963, estaban activos 120 hermanamientos y hoy en día se cuentan más de 40.000 acuerdos locales (CEMR). A finales de los cincuenta también las regiones comenzaron a forjar acuerdos con sus vecinas. En 1958 se estableció, a lo largo de la frontera entre Alemania y Holanda, la primera eurorregión, llamada Euregio (o Eurorregión Gronau), que representa un precedente para la institucionalización de muchas Eurorregiones. Hasta los años noventa, eran especialmente activas las regiones de Europa centro-occidental y septentrional. Con la entrada en vigor del Tratado de Schengen y la eliminación progresiva de barreras para la libre circulación de mercancías, personas y capitales, también la zona mediterránea empezó a moverse en esta dirección (Perkmann, 2003; Husák, 2010). La ampliación de la Unión Europea hacia el este ha conducido, a lo largo de los años 2000, a un crecimiento exponencial de las regiones transfronterizas tanto a lo largo de las fronteras exteriores de la UE como entre los estados miembros. Además, las autoridades locales y regionales disponen, a través de la $\mathrm{AECT}$, de una nueva herramienta no solamente limitada a la gestión de los programas Interreg y a los fondos estructurales, sino que representa un impulso hacia una nueva gobernanza del territorio (Sousa, 2013; Evrad, 2016).

\footnotetext{
bros de la misma pueden ser autoridades nacionales, regionales y locales (de al menos dos estados miembros), que tendrán que aprobar una Convención, una Constitución y elegir la sede decidiendo qué leyes nacionales aplicarán en caso de necesidad. Las competencias han de estar bien delimitadas para evitar conflictos con las autoridades nacionales. Finalmente, las principales responsabilidades se refieren a facilitar y a promocionar la cooperación territorial a la luz de la cohesión económica y social. Más específicamente, tiene la función de facilitar la implementación de los proyectos de cooperación financiados tanto por los fondos estructurales como por otras entidades públicas.
} 
Tabla 2. Estructuras de cooperación transfronterizas activas entre España y Francia, subrayando las activas en la Cerdaña (iniciativas bottom-up)

\begin{tabular}{|c|c|c|c|c|c|}
\hline Nombre & $\begin{array}{l}\text { Año de institución } \\
\text { (estatus AECT/AEIE/ } \\
\text { Consorcio) }\end{array}$ & $\begin{array}{l}\text { Estatus } \\
\text { jurídico }\end{array}$ & $\begin{array}{l}\text { Jurisdicción } \\
\text { aplicada }\end{array}$ & Escala & $\begin{array}{c}\text { Área } \\
\text { geográfica }\end{array}$ \\
\hline $\begin{array}{l}\text { Comisión Internacional } \\
\text { de los Pirineos (CIP) }\end{array}$ & 1875 & No previsto & No previsto & Internacional & Pirineos \\
\hline $\begin{array}{l}\text { Comunidad de Trabajo } \\
\text { de los Pirineos (CTP) }\end{array}$ & 1983 (2005) & Consorcio & Española & Suprarregional & Pirineos \\
\hline $\begin{array}{l}\text { AECT Eurorregión Nueva } \\
\text { Aquitania-Euskadi-Navarra }\end{array}$ & $1982(2011)$ & AECT & Francesa & Regional & Occidental \\
\hline $\begin{array}{l}\text { AECT Eurorregión Pirine- } \\
\text { os-Mediterránea }\end{array}$ & 2004 (2009) & AECT & Francesa & Regional & Oriental \\
\hline Espacio Pourtalet & 2011 & AECT & Española & Regional & Central \\
\hline Huesca-Haute Pyrénées & 2014 & AECT & Española & Supralocal & Central \\
\hline $\begin{array}{l}\text { Eurodistrito del Espacio } \\
\text { Catalán Transfronterizo }\end{array}$ & 2007 & $\begin{array}{l}\text { Declaración } \\
\text { de intención }\end{array}$ & No previsto & Supralocal & Oriental \\
\hline Eurociudad Vasca & 1993 (1997) & AEIE & Española & Local & Occidental \\
\hline Consorcio Bidasoa-Txingudi & 1998 & Consorcio & Española & Local & Occidental \\
\hline AECT Pirineos-Cerdaña & $\underline{2011}$ & $\underline{\mathrm{AECT}}$ & Francesa & Local & $\underline{\text { Oriental }}$ \\
\hline $\begin{array}{l}\text { AECT Hospital } \\
\text { de la Cerdaña }\end{array}$ & $\underline{2010}$ & AECT & Española & Local & Oriental \\
\hline $\begin{array}{l}\text { AECT 'País de Arte y de } \\
\text { Historia Transfronterizo - } \\
\text { Los Valles Catalanas del Tec } \\
\text { y del Ter' }\end{array}$ & 2015 & AECT & Francesa & Local & Oriental \\
\hline
\end{tabular}

Fuente: elaboración propia a partir de Sanguin (2013).

La frontera entre España y Francia constituye hoy en día un laboratorio de gobernanza transfronteriza muy interesante (Latamendia, 2011). A lo largo de los Pirineos se cuentan doce estructuras de cooperación activas en diferentes escalas geográficas (tabla 2): en el conjunto del área transfronteriza actúa desde 1983 la Comunidad de Trabajo de los Pirineos (CTP), que reúne todas las regiones fronterizas pirenaicas con la finalidad de cooperar para el desarrollo socioeconómico, cultural y para la preservación medioambiental del macizo montañoso. Desde el año 2005 dicha cooperación se ha formalizado en un consorcio (bajo la jurisdicción española), donde participa también el microestado de Andorra. La CTP ha sido designada como Autoridad de Gestión del Poctefa 2007-2013 y de la actual programación Interreg Poctefa 2014-2020. A escala regional, la Eurorregión Nueva Aquitania-Euskadi-Navarra (EAEN) y la Eurorregión Pirineos-Mediterráneo (EPM) estructuran sus estrategias más allá de los programas comunitarios, proponiendo estrategias integradas de desarrollo Eurorregional (EAEN) o bien ambiciosos proyectos temáticos (EPM). Las estructuras de cooperación locales reflejan, en cambio, la necesidad de resolver 
conjuntamente las problemáticas específicas de cada territorio (la gestión del litoral vasco, el desarrollo rural en la Cerdaña, etc.) a través de distintos sistemas de gobernanza. En la tabla 2 quedan reflejadas las estructuras actualmente vigentes. La gran mayoría (casi el 60\%) han adoptado el estatus de AECT para superar los obstáculos burocráticos a la cooperación, por un lado, y para captar los fondos europeos de manera más eficaz, por el otro. Finalmente hay un nivel intermedio, que definimos supralocal, donde las instituciones locales se unen para desarrollar unas estrategias de desarrollo territorial y socioeconómico. Las dos iniciativas presentes en el Pirineo Central y Oriental han nacido como proyectos del Poctefa 2007-2013. La AECT Huesca-Haute Pyrénées es ya una estructura formalizada, mientras que el Eurodistrito del Espacio Catalán Transfronterizo no ha conseguido todavía todo el respaldo político-institucional necesario de los gobiernos centrales (Castañer y Feliu, 2013) y queda como un espacio de cooperación apoyado por un fondo de microproyectos entre la Generalitat de Cataluña y el Conseil Général des Pyrénées Orientales.

\section{3. ¿Vamos hacia una integración territorial transfronteriza?}

A partir de lo analizado hasta el momento, se nos hace evidente que los agentes territoriales interpretan la cooperación transfronteriza como una estrategia territorial complementaria de desarrollo socioeconómico, paralela a las políticas tradicionales (por ejemplo las políticas regionales y nacionales). Citando a Sohn (2014), la pregunta es la siguiente: estas nuevas entidades jurídicoadministrativas ¿están capacitadas para alcanzar una integración territorial del espacio transfronterizo?

La región pirenaica tiene un fuerte carácter rural (excluyendo el litoral vasco y los pasos fronterizos como el de la Jonquera-Le Perthus). Estos espacios presentan, a menudo, una condición socioeconómica desfavorecida, de despoblamiento y de falta de infraestructuras y servicios. Sin embargo, las sociedades locales han sabido históricamente beneficiarse, a partir de diferentes mecanismos, de la presencia de la frontera (Sahlins, 1989; Brunet, 2002); por ejemplo, a través de las relaciones comerciales legales o ilegales (contrabando) entretejidas entre las comunidades fronterizas. La identidad local y las históricas relaciones de proximidad entre las comunidades locales constituyen dos importantes factores clave de la CTF local. Por lo tanto, la cooperación transfronteriza local puede representar una estrategia eficaz para aumentar la cohesión institucional, proporcionar los servicios y las infraestructuras necesarias, valorizando el patrimonio cultural y natural compartido (Berzi, 2016). En este escenario, la gobernanza multinivel representa un elemento fundamental para garantizar la sostenibilidad temporal y la eficacia de la cooperación (Morata, 2004). A lo largo de la frontera entre España y Francia se están configurando nuevas perspectivas y oportunidades para la gestión del territorio transfronterizo. Ejemplos de ello son el área funcional sanitaria de la Cerdaña (Berzi y Durà, 2019), los distritos turísticos integrados (Blasco et al., 2014), las estrategias para el espacio urbano-litoral vasco o las áreas protegidas transfronterizas (Bénos et al., 2007). En los siguientes apartados consideramos el caso de la Cerdaña. 


\section{Metodología}

El estudio de caso se fundamenta en la investigación empírica llevada a cabo entre 2013 y 2015 en la Cerdaña, utilizando las siguientes fuentes de datos y métodos: a) las referencias académicas, como las investigaciones de Sahlins (1989), Mancebo (1999), Moncusí (2005), Oliveras (2013), etc. b) las fuentes institucionales de información estadística (IDESCAT; INSEE), $c$ ) el análisis detallado de los Programas de Cooperación Transfronteriza (Poctefa 2007-2013; Interreg IIIA, 2000-2006; Interreg IIA 1994-1999, e Interreg I $1989-1993 ;$ d) las entrevistas semiestructuradas presenciales a actores locales y regionales (tanto de naturaleza pública y privada), que han tenido y tienen un papel clave en la CTF, y e) la consulta de fuentes locales.

En las entrevistas a actores locales y regionales intentábamos descifrar, a partir de sus relatos, la incubación de los proyectos, el liderazgo de los mismos, las estrategias desarrolladas, las redes de gobernanza establecidas y, finalmente, su grado de implementación, gestión y viabilidad real. De las entrevistas realizadas destacamos los relatos de los alcaldes, del presidente del Consejo Comarcal de la Cerdaña (Baja Cerdaña) y del gerente y director médico del Hospital de la Cerdaña.

La información ha sido catalogada y procesada a partir de dos bases de datos con la finalidad de identificar, por un lado, los factores más importantes de la CTF local (históricos, socioeconómicos y culturales) y, por otro, para analizar los recientes proyectos de cooperación transfronteriza. En el apartado 4.3 analizamos tres de las iniciativas más significativas, concentrándonos, sobre todo, en los resultados de los proyectos y en la gobernanza territorial.

\section{Caso de estudio de la Cerdaña (análisis y resultados)}

La Cerdaña representa un área de estudio ejemplar para analizar el papel de la cooperación transfronteriza en el proceso de integración funcional y espacial. Para su estudio, en primer lugar vamos a constatar como la uniformidad del territorio, las similitudes del sistema socioeconómico y los vínculos culturales, antes y después de la aparición de la frontera, constituyen unos factores importantes que están en la base de la cooperación transfronteriza. En segundo lugar analizaremos la institucionalización de la cooperación, subrayando su evolución y su estabilidad temporal. Finalmente, destacamos tres proyectos emblemáticos para la integración funcional: el Hospital de la Cerdaña, el futuro matadero compartido y el plan del paisaje transfronterizo.

\subsection{Un territorio eminentemente transfronterizo}

La Cerdaña es una microrregión de unos $1000 \mathrm{~km}^{2}$ situada en los Pirineos orientales. Desde el Tratado de los Pirineos de 1659, que delimita la frontera entre España y Francia, el valle queda dividido en dos partes: la Alta Cerdaña, en Francia (región Occitanie Pyrénées-Méditerranée), que pertenece al Canton 
Figura 1. Ubicación de la Cerdaña en el marco pirenaico
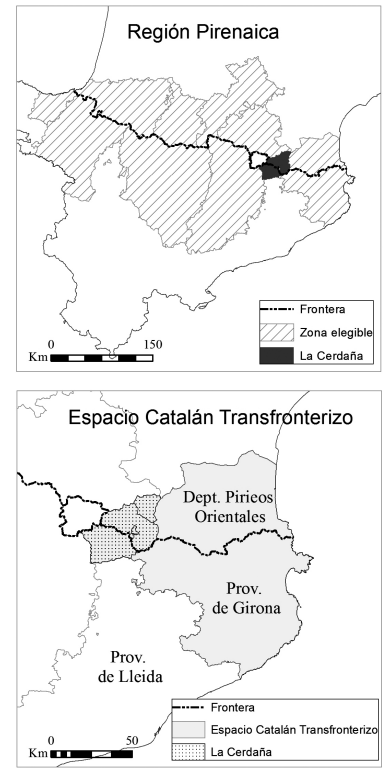

Fuente: elaboración propia.

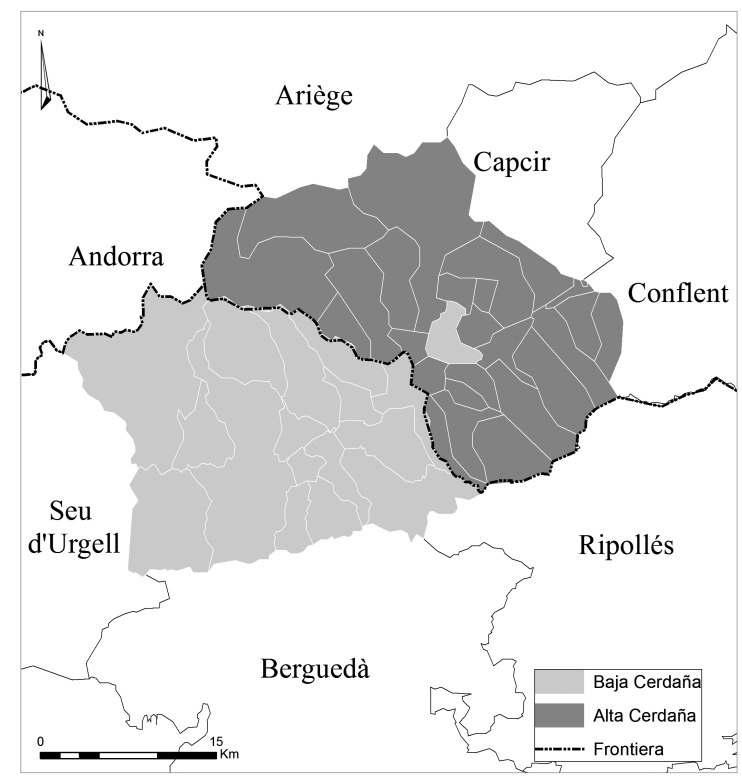

des Pyrénées catalanes, y la Baja Cerdaña, en España (Comunidad Autónoma de Cataluña), repartida entre la provincia de Girona y la provincia de Lleida (figura 1).

La unidad geográfica, histórica y cultural ha sido remarcada en muchas investigaciones geográficas (Vila, 1984[1926]; Tulla, 1977, 1997; Mancebo, 1999), históricas (Sanabre, 1960; Sahlins, 1989; Blanchon, 1992) y antropológicas (Moncusí, 2005), en las cuales la frontera aparece como un elemento artificial. El medio físico está compuesto por una llanura de $200 \mathrm{~km}^{2}$ rodeada por unos macizos montañosos que la encierran casi totalmente, distinguiéndola claramente de los territorios circundantes. La Cerdaña es rica en zonas boscosas, prados y está atravesada por los ríos Segre y Carol, recursos que han sido, a la vez, tema de conflictos y de iniciativas de la CTF (Oliveras, 2013). Actualmente, la zona cuenta con unos 31.000 habitantes, siendo más numerosos los que habitan en la Baja Cerdaña (IDESCAT; INSEE). Los pequeños asentamientos urbanos están ubicados principalmente en el valle y el principal centro socioeconómico de referencia es la ciudad de Puigcerdà. La sociedad ceretana ha sido históricamente rural, con la ganadería y la agricultura como actividades fundamentales, las cuales generaron el típico paisaje local (bocage). La pequeña industria de transformación local como mataderos y los establecimientos textiles se concentraban principalmente en Puigcerdà (Vila, 1984[1926]).

El relativo aislamiento geográfico, por un lado, y el hecho de compartir un espacio y una cultura catalana común, por otro, constituyen factores impor- 
tantes que son la base de las relaciones de proximidad y de la identidad local. Ya antes de la aparición de la frontera, las autoridades locales mantuvieron una institución política y administrativa local, el Consejo General de los Síndicos (Sahlins, 1989), que regulaba la explotación de recursos naturales (aguas, pastos y bosques). De hecho, Sahlins recuerda como la Cerdaña era, a principios del siglo XVIII, una de las comarcas más unificadas de toda Cataluña (ibid.) que, en principio, no fue interrumpida por la división política. Hasta mediados del siglo XIX, el matrimonio, la propiedad y las relaciones económicas entre la Alta y la Baja Cerdaña eran muy entretejidas, y varias actividades legales e ilegales (el contrabando) florecían a ambos lados de este territorio (Moncusí, 2005). A partir de aquel momento, las diferentes políticas de los estados espanol y francés en temas de infraestructuras y fiscales generaron un proceso de diferenciación socioeconómica, cultural e incluso mental (Sahlins, 1989). La Alta Cerdaña, mejor conectada con el interior de Francia, empezó primero a desarrollar el turismo invernal, mientras que la parte española se mantuvo más agrícola (la carretera Puigcerdà-Ripoll no fue construida hasta el año 1914 y el ferrocarril llegó en 1922). También el paisaje empezó a modificarse, a causa de la modernización de la agricultura y el desarrollo del sector terciario (especialmente por el turismo de invierno y las segundas residencias de baja densidad para las élites barcelonesas). Las relaciones transfronterizas históricas entre las instituciones locales fueron drásticamente reducidas entre 1850 y 1980 y delegadas al gobierno nacional (Oliveras, 2013). Específicamente, la permeabilidad de la frontera se redujo significativamente entre 1939 y 1947 , como consecuencia de la instauración de la dictadura española.

\subsection{Una cooperación institucional estable}

El proceso de integración europea ha producido un empoderamiento de las instituciones locales, las cuales disponen hoy de un importante know-how en temas de cooperación transfronteriza. La continuidad y la densidad de iniciativas locales son un caso único en el Pirineo Oriental y, junto con el litoral vasco, en el conjunto de los Pirineos.

En la Cerdaña, la CTF se encuentra entre las instituciones con más experiencia, algunas de las cuales se remontan a finales de los ochenta del siglo anterior, cuando España entró en el Mercado Común de la Comunidad Europea. La apertura de la frontera hispano-francesa implicó un cambio de percepción por parte de las administraciones locales, antes mucho más reticentes a cooperar (Mancebo, 1999). En la tabla siguiente resumimos las principales iniciativas detectadas en la literatura y también desde la prensa local (tabla 3). Las asociaciones culturales fueron importantes impulsoras de la cooperación institucional. Destacamos el Institut d'Estudis Ceretans, una asociación que promueve la visión de una Cerdaña geográficamente, culturalmente y políticamente unida. Desde 1981 dicha entidad organiza muchas iniciativas transfronterizas, entre las cuales destaca la «Diada de la Cerdaña», un encuentro anual de carácter lúdico, festivo y político que se celebra alternativamente en un pueblo de la 
Tabla 3. Principales iniciativas de cooperación transfronteriza institucional en la Cerdaña desde los años ochenta

\begin{tabular}{|c|c|c|c|c|c|}
\hline Iniciativa & Año & Promotor & Participantes & Finalidad & $\begin{array}{l}\text { Evolución/ } \\
\text { estado actual }\end{array}$ \\
\hline Diada de la Cerdaña & 1982 & $\mathrm{IEC}$ & Municipios Cerdaña & $\begin{array}{l}\text { Política y } \\
\text { lúdico-recreativa }\end{array}$ & Activa \\
\hline $\begin{array}{l}\text { Manifiesto de Cooperación } \\
\text { Interfronteriza }\end{array}$ & 1989 & Llívia & $\begin{array}{l}\text { Estavar, Angoustrine- } \\
\text { Villeneuve-des-Escaldes, } \\
\text { Sainte-Léocadie, Bourg- } \\
\text { Madame-la-Guingueta } \\
\text { d'Hix }\end{array}$ & Proyectos Interreg & $\begin{array}{l}\text { Carta de coopera- } \\
\text { ción Llívia-Estavar }\end{array}$ \\
\hline $\begin{array}{l}\text { Asociación de Municipios } \\
\text { de la Cerdaña }\end{array}$ & 1991 & IEC & 43 sobre 50 municipios & Política & $\begin{array}{l}\text { Manifiesto de } \\
\text { Font-romeu }\end{array}$ \\
\hline $\begin{array}{l}\text { Carta de cooperación } \\
\text { Llívia-Estavar }\end{array}$ & 1991 & Estavar & Llívia & Cultural & Activa \\
\hline $\begin{array}{l}\text { Consorcio Puigcerdà- } \\
\text { Bourg-Madame (I) }\end{array}$ & 1995 & Puigcerdà & Bourg-Madame & $\begin{array}{l}\text { Ordenación del } \\
\text { territorio }\end{array}$ & $\begin{array}{l}\text { Consorcio } \\
\text { Puigcerdà - } \\
\text { Bourg-Madame } \\
\text { (II) }\end{array}$ \\
\hline $\begin{array}{l}\text { Manifiesto de } \\
\text { Font-romeu }\end{array}$ & 1998 & AMC & Municipios Cerdaña & $\begin{array}{l}\text { Unidad política } \\
\text { local }\end{array}$ & $\begin{array}{l}\text { Gran Consejo } \\
\text { de la Cerdaña }\end{array}$ \\
\hline $\begin{array}{l}\text { Consejo General de } \\
\text { la Cerdaña }\end{array}$ & 2009 & $\begin{array}{l}\text { IEC } \\
\text { (alcaldes) }\end{array}$ & 30 municipios & $\begin{array}{l}\text { Unidad política } \\
\text { local }\end{array}$ & $\begin{array}{l}\text { AECT } \\
\text { Pirineos-Cerdaña }\end{array}$ \\
\hline $\begin{array}{l}\text { AECT Pirineos- } \\
\text { Cerdaña }\end{array}$ & 2011 & $\begin{array}{l}\text { Consejo Comarcal } \\
\text { de la Cerdaña - } \\
\text { Communauté de } \\
\text { Communes Pyré- } \\
\text { nées Cerdagne }\end{array}$ & Los promotores & $\begin{array}{l}\text { Turismo } \\
\text { — Desarrollo eco- } \\
\text { nómico local } \\
\text { — Valorización del } \\
\text { patrimonio } \\
\text { histórico y } \\
\text { natural }\end{array}$ & Activa \\
\hline $\begin{array}{l}\text { Consorcio Puigcerdà- } \\
\text { Bourg-Madame (II) }\end{array}$ & 2015 & Puigcerdà & Bourg-Madame & $\begin{array}{l}\text { Cultura } \\
\text { Deporte } \\
\text { Turismo } \\
\text { Servicios sociales } \\
\text { Urbanismo }\end{array}$ & Reactivado \\
\hline
\end{tabular}

Fuente: elaboración propia a partir de Mancebo (1999) y Oliveras (2013).

Alta y de la Baja Cerdaña (Oliveras, 2013). Durante estas jornadas, los participantes han propuesto muchas iniciativas de cooperación como la creación de la Asociación de Municipios de la Cerdaña (AMC) que, en 1991, agrupaba un elevado número de municipios (43 de 50 totales) y promovía iniciativas conjuntas en sectores como el turismo, el medio ambiente, las infraestructuras, los transportes, la salud y la cultura. Hacia finales de los años noventa, durante una Diada fue aprobado el Manifiesto de Font-Romeu, en el cual se recogen la creación del Consejo General de la Cerdaña para reunir el Consejo Comarcal de la Cerdaña y la Communauté de Communes Pyrénées-Cerdagne en una misma organización. Esta iniciativa se concretó en 2011 con la creación 
de la AECT Pirineos-Cerdaña, compuesta por dichas instituciones. Desde el año 2009, la Diada cuenta con una nueva bandera que, simbólicamente, une a las dos partes. Es interesante notar como las estrategias bilaterales entre ayuntamientos (one-to-one), después de un periodo de inactividad, han vuelto recientemente a reactivarse. Es el caso de la cooperación entre Llívia y Estavar que, después del primer intento de cooperación a principios de los noventa, han vuelto a proponer un festival de cine transfronterizo y están elaborando un proyecto de hermanamiento entre los dos municipios. En 2016, también, los ayuntamientos de Puigcerdà y de Bourg-Madame, representantes de una única aglomeración urbana a lo largo de la frontera, han reactivado un consorcio de 1995 para promover iniciativas culturales y para coordinar el desarrollo urbano.

\section{3. ¿Hacia un proyecto territorial transfronterizo?}

Las instituciones locales, junto con las autoridades regionales y nacionales, han activado proyectos transfronterizos ambiciosos y de envergadura. Los han activado a partir de la financiación comunitaria y de recursos propios. Seguidamente, presentamos tres iniciativas que, según nuestra hipótesis, configurarán una Cerdaña económicamente, socialmente y territorialmente más integrada.

\subsubsection{Desde el hospital hacia un distrito sanitario transfronterizo}

La cooperación transfronteriza en materia de salud es hoy en día un tema muy emblemático tanto para la Cerdaña como para Europa (Berzi y Durà, 2019). Históricamente, el único hospital del valle se encontraba en Puigcerdà y dicha infraestructura no era utilizada por los habitantes de la Alta Cerdaña por dos razones. Primera, por la falta de convenios entre las autoridades responsables (CatSalut en Cataluña y el Ministerio de Sanidad francés), y segunda, por la histórica desconfianza de los ciudadanos franceses hacia la sanidad española, considerada cualitativamente inferior (Moncusí, 2005). Por lo tanto, el hospital francés de referencia se encontraba en Prades $(65 \mathrm{~km})$ o en Perpiñán $(100 \mathrm{~km})$.

Como documenta Oliveras (2013), los primeros intentos de cooperación se remontan a principios de los noventa como respuesta a los efectos negativos causados por la reorganización del sistema sanitario francés y español. Además, algunos acontecimientos fortuitos y extraordinarios (en 1996 y 2001 la conexión entre la Alta Cerdaña y Prades se cortó debido a un deslizamiento de tierra y a una fuerte nevada), indujeron a los ceretanos franceses a utilizar el Hospital de Puigcerdà. Surgió entonces, a principio de 2000, la idea de construir un nuevo hospital en común. En 2003 se financió, a través del Interreg IIIA, un estudio de viabilidad, el resultado positivo del cual condujo, en 2009, al proyecto ejecutivo (promovido por la Generalitat de Cataluña y el Ministerio de Sanidad francés), que obtuvo $18 \mathrm{M} €$ del FEDER dentro de la programación Poctefa 2007-2013.

La gestión del futuro hospital fue encomendada a la nueva Fundación Hospital de la Cerdaña (compuesta por los promotores del proyecto ejecutivo), 
que adoptó en 2010 el estatuto de AECT. El nuevo marco legal y político está contribuyendo a simplificar muchas cuestiones burocráticas y legales, tanto de carácter sanitario (diferentes regímenes de gestión) como administrativo (registro de nacimientos y defunciones). La gobernanza del hospital tiene un enfoque multinivel e involucra las instituciones locales en la toma de decisiones (en el consejo de administración encontramos el Consejo Comarcal de la Cerdaña y el Ayuntamiento de Puigcerdà; mientras que en el consejo consultivo hay las administraciones locales de ambas partes).

Desde el mes de septiembre de 2014, la infraestructura está en funcionamiento y la población francesa atendida sigue creciendo (AECT Hospital de la Cerdanya, 2016). Además, el hospital es un centro de referencia europeo para la medicina de montaña (sobre todo rescate y traumatología). Más allá de la infraestructura en sí, un reto ambicioso es la puesta en marcha de un área sanitaria transfronteriza; es decir, la coordinación y la puesta en red de los servicios públicos y privados (centros médicos y de deporte, residencias de la tercera edad, etc.). Un resultado concreto ha sido el recién traslado de la unidad de emergencia francés (SIVU) al hospital transfronterizo. Por primera vez, una unidad francesa sanitaria opera fuera del territorio nacional. Parece por tanto que el hospital transfronterizo está reduciendo el efecto de las barreras mentales, psicológicas y socioculturales que aún existen de la parte francesa hacia la asistencia sanitaria española, y que está tomando forma un área sanitaria transfronteriza.

\subsubsection{El apoyo al sector cárnico}

La ganadería y el sector cárnico representan, junto con la agricultura, las actividades económicas tradicionales de la Cerdaña. Desde los años setenta, la crisis del sector agropecuario ha afectado la rentabilidad de la producción de la comarca (Oliveras, 2012). En los años ochenta, la reforma técnico-sanitaria de los mataderos indujo a la reorganización del sector en ámbitos supralocales. Muchas estructuras cerraron y la producción se concentró casi únicamente en los mataderos de Puigcerdà y de Bellver (Baja Cerdaña) y en el de Bourg-Madame (Alta Cerdaña). Sin embargo, esta estrategia no fortaleció la producción cárnica local porque casi la totalidad de las reses se sacrificaban fuera de la comarca (la Seu d'Urgell) o en localidades alejadas a más de $100 \mathrm{~km}$ (Girona, Narbona o Perpiñán). Durante la Diada de la Cerdaña de 2002, el Consejo Comarcal de la Cerdaña y la organización de productores cárnicos francesa (el Syndicat de l'Abattoir Cerdagne-Capcir, SACC) presentaron la idea de un nuevo matadero compartido por todo el valle. En 2006 se realizó un estudio de viabilidad financiado por el Interreg IIIA, cuyo resultado positivo condujo a presentar el proyecto ejecutivo en el Poctefa 2007-2013, el cual recibió casi 2M € del FEDER. El proyecto MTC - Matadero Transfronterizo de la Cerdaña está coordinado por el alcalde de Estavar (también vicepresidente de la Communauté de Communes Pyrénées Cerdagne). La construcción del equipamiento empezó en 2011 y finalizó 
en 2017. La falta de recursos (por parte española) retrasó la ejecución de las obras. El objetivo es, por un lado, fomentar la producción cárnica local, con una estimación mediana de 800 toneladas/año y, por otro, cerrar el ciclo de producción dentro de la comarca. Además, los promotores están estudiando una certificación de calidad propia compartida, para valorizar sus productos en los mercados extralocales. Un valor añadido es la posibilidad que tendrán los ganaderos de vender sus propios productos a distribuidores minoristas. Para gestionar la estructura se ha creado el Syndicat intercommunal de l'abattoir de Cerdagne Capcir Haut-Conflent que, bajo la legislación francesa, se transformará en una Sociedad Cooperativa de Interés Colectivo (SCIC), que reunirá el Consejo Comarcal de la Cerdaña, el Sindicato intercomunal, las agrupaciones de ganaderos, los carniceros y mayoristas. Análogamente al Hospital de la Cerdaña, no hay un precedente entre los dos países y se tendrán que resolver muchas cuestiones legales.

\subsubsection{El Pla del Paisatge como estrategia de integración territorial transfronteriza}

El paisaje de la Cerdaña ha experimentado, durante las últimas décadas, una evolución importante. La terciarización de la comarca, especialmente debido al desarrollo del sector de la construcción y el turismo, ha conllevado a la aparición de segundas residencias y la pérdida sustancial del paisaje productivo que tenía el bocage ceretano. En la 31 a Diada de la Cerdaña (celebrada en 2013 en Portes, Alta Cerdaña), las instituciones locales propusieron, bajo la AECT Pirineos-Cerdaña, la elaboración de un catálogo de paisaje de toda la comarca, sin tener en cuenta los límites administrativos. El proyecto tiene el objetivo de promover una oferta turística (y económica en general) basada en criterios geográficos y paisajísticos. El catálogo, elaborado por el Observatorio del Paisaje de Cataluña y por el Parque Regional Francés del Pirineo Catalán, constituye una herramienta de planificación estratégica para promover un desarrollo local virtuoso basado en los valores estéticos del paisaje (Observatori del Paisatge de Catalunya, 2013) y se dirige a agentes locales económicos, sociales e institucionales del valle.

En 2013 se constituyó un Comité Piloto y se realizaron varias reuniones con los actores locales. La elaboración del plan está todavía en curso, pero ya se han logrado algunos resultados importantes. Por ejemplo, se han identificado siete objetivos comunes de calidad paisajística y disponen de un glosario común de paisajes, una cartografía compartida y un manual específico de conservación del paisaje. En 2014-2015 se ha llevado a cabo una consulta pública para mejorar el proceso participativo y dos seminarios formativos. El plan de paisaje debería permitir al gobierno local superar la divergencia de los sistemas de planificación francés y catalán para integrar la estrategia común en sus propias herramientas de planificación. Esta iniciativa podría conducir, a largo plazo, a una efectiva planificación territorial transfronteriza. 


\section{Conclusiones}

En las últimas décadas, los cambios en la geopolítica mundial y europea han situado las áreas de frontera en el centro de la discusión institucional y académica. Por un lado, los border studies han contribuido tanto analíticamente como empíricamente a un mejor conocimiento de las dinámicas sociales, económicas, políticas que se generan en dichas áreas. Por el otro, la evolución de las políticas comunitarias (top-down) ha fomentado iniciativas locales de cooperación entre los agentes territoriales (bottom-up). En este sentido, los Pirineos constituyen un área de estudio privilegiada. Desde finales de los ochenta, a lo largo de la frontera hispano-francesa se han ejecutado muchas iniciativas de cooperación que, a menudo, han generado sinergias entre los actores locales, regionales y nacionales. Estos vínculos han conllevado, en el caso de las instituciones públicas, a la creación de organismos de cooperación estables. La gobernanza multinivel resulta ser un enfoque fundamental: las escalas territoriales interactúan tanto verticalmente (desde el nivel comunitario hasta el nivel regional y local) como horizontalmente: los actores regionales y locales participan de manera proactiva, proponiendo proyectos y acciones intersectoriales y generando redes de cooperación.

La microrregión transfronteriza de la Cerdaña representa un caso ejemplar de área periférica montañosa europea. A través del análisis de la misma hemos demostrado como la especificidad geográfica, las relaciones históricas de vecindad, la fuerte identidad local y el hecho de compartir retos comunes, son factores fundamentales para el desarrollo de una CTF exitosa, estable, multitemática y de largo plazo. Las tres iniciativas analizadas (el hospital, el matadero y el plan del paisaje) representan una respuesta conjunta a la falta de servicios, de infraestructuras y de políticas adecuadas para las áreas de frontera periféricas, beneficiándose del apoyo legal y financiero proporcionado por la UE. La CTF está generando unos nuevos «vínculos» sociales, económicos y políticos que están induciendo a una fuerte interdependencia territorial entre la Alta y la Baja Cerdaña. De esta manera, es posible reducir las fronteras mentales y armonizar las asimetrías institucionales que todavía constituyen dos de los obstáculos más importantes para una efectiva integración territorial. Desde esta perspectiva se considera que los resultados se podrían extrapolar a otras áreas montañosas fronterizas de la UE, como los Alpes, los Cárpatos o la región balcánica. Es posible hipotetizar, por lo tanto, una "vía transfronteriza al desarrollo local», en la que los agentes territoriales, tanto los públicos como los privados, elaboren acciones y políticas donde la frontera no representa un límite al desarrollo, sino un recurso estratégico común.

\section{Referencias bibliográficas}

AECT Hospital de la Cerdanya (2016). Memòria - Rapport Annuel 2016. <http:// www.hcerdanya.eu/sites/default/files/media/media/Documents/memoria_rapport_ annuel_2016.pdf> [Consulta: 10 de mayo de 2017]. 
Alland, A. y Alland, S. (2006). Catalunya, one nation, two states: an ethnographic study of nonviolent resistance to assimilation. Nueva York: Palgrave MacMillan.

Allen, D. (2010). "The structural funds and cohesion policy». Policy-making in the European Union (6), 229-251.

Berzi, M. (2013). "Cross-border Cooperation and Local Development in the Pyrenees. The case of Cerdanya». European Journal of Geography, 4 (4), 47-60.

- (2016). «La cooperación transfronteriza como soporte al desarrollo local endógeno en las áreas de montaña: el caso de la Cerdanya». En: J. M. TRILlo y I. Pires (eds.). Fronteras en la investigación peninsular: temáticas y enfoques contemporáneos/Fronteiras na investigação peninsular. Santiago de Compostela: Servizo de Publicacións e Intercambio Científico, 49-68.

- (2017). "The cross-border reterritorialization concept revisited: the territorialist approach applied to the case of Cerdanya on the French-Spanish border», European Planning Studies, 1-22. <https://doi.org/10.1080/09654313.2017.1321622>

BERZI, M. y DurÀ, A. (2019). "La coopération transfrontalière en matière sanitaire dans l'UE à travers le cas emblématique de l'Hôpital de Cerdagne (Pyrénées)». En: F. Moullé (ed.). Maillages et interfaces, les enjeux territoriaux de la santé. Arras: Artois Presses Université (en prensa).

Bénos, R.; Cazenave-Piarrot, A. y Milian, J. (2007). «Pyrenees-Mont Perdu Patrimoine Mondial : un espace montagnard a l'epreuve de la protection et de sa gestion». En: Pyrenees-Mont Perdu Patrimoine Mondial: un espace montagnard a l'epreuve e la protection et de sa gestion. Espagne: BAEZA, 47-63. <https://halshs. archives-ouvertes.fr/hal-00374871> [Consulta: 07 de novembre de 2016].

BlanCHON, J. (1992). La Cerdagne pays-frontière: 1936-1948, tesis doctoral no publicada. Toulouse: Université Toulouse 2.

Blasco, D.; GuiA, J. y Prats, L. (2014). "Tourism destination zoning in mountain regions: a consumer-based approach». Tourism Geographies, 16 (3), 512-528.

Brunet, S. (2002). "Les mutations des lies et passeries des Pyrénées, du XIVe au XVIIIe siècle». Annales du Midi : revue archéologique, historique et philologique de la France méridionale, 114 (240), 431-456. $<$ https://doi.org/10.3406/anami.2002.2784>

CASTAÑER, M. y Feliu, J. (2013). «L'eurodistricte català transfronterer. Un espai emergent sense marc administratiu». Treballs de la Societat Catalana de Geografia, 74, 41-58.

Centro Superior de Estudios de la Defensa Nacional (CESEDEN) (1991). Los transportes y la barrera pirenaica. Madrid: Ministerio de Defensa.

Council of Europe. Protocol No. 3 to the European Outline Convention on Transfrontier Co-operation between Territorial Communities or Authorities concerning Euroregional Co-operation Groupings (ECGs). CETS No. 206 - Utrecht, 16.XI.2009.

- Protocol No. 2 to the European Outline Convention on Transfrontier Co-operation between Territorial Communities or Authorities concerning interterritorial co-operation. ETS No. 169 - Strasbourg, 05.V.1998.

- Additional Protocol to the European Outline Convention on Transfrontier Co-operation between Territorial Communities or Authorities. ETS No. 169 - Strasbourg, 09.XI.1995.

- European outline convention on transfrontier co-operation between territorial communities or authorities. ETS No. 106 - Madrid, 21.V.1980. 
Decoville, A.; Durand, F.; Sohn, C. y Walther, O. (2013). "Comparing cross-border metropolitan integration in Europe: towards a functional typology». Journal of Borderlands Studies, 28 (2), 221-237. <https://doi.org/10.1080/08865655.2013.854654>

DufFy, R. (2006). "The potential and pitfalls of global environmental governance: The politics of transfrontier conservation areas in Southern Africa». Political Geography, 25 (1), 89-112.

Engl, A. (2007). «Future Perspectives on Territorial Cooperation in Europe: The EC Regulation on a European Grouping of Territorial Cooperation and the Planned Council of Europe Third Protocol to the Madrid Outline Convention concerning Euroregional Co-operation Groupings». European Diversity and Autonomy Papers - EDAP, 3. <www.eurac.edu/edap> [Consulta: 6 de diciembre de 2016].

European Commission. Directorate-General for Regional and Urban Policy (2016), Collecting solid evidence to assess the needs to be addressed by Interreg cross-border cooperation programmes (2015CE160AT044). Final Report. <http://ec.europa.eu/regional_policy/sources/docgener/studies/pdf/cross_border/border_regions_final_report.pdf> [Consulta: 7 de diciembre de 2016].

Evrard, E. (2016). "The European Grouping of Territorial Cooperation (EGTC): Towards a Supraregional Scale of Governance in the Greater Region SaarLorLux?». Geopolitics, 1-25. $<$ https://doi.org/10.1080/14650045.2015.1104667>

FERnÁNDEZ-SÁNCHEZ, P. A. (ed.) (2008). La asimetría institucional entre España y Portugal en el marco de la cooperación transfronteriza (Andalucía, Algarve y Alentejo). Barcelona: Atelier Libros.

Feliu, J.; Berzi, M.; Vicente, J.; Castañer, M. y Llussà, R. (2013). «Análisis de los proyectos y actores transfronterizos España-Francia en el periodo 20072013». Geographicalia, 63, 75-93.

Haller, D. (2013). Gelebte Grenze Gibraltar: Transnationalismus, Lokalität und Identität in kulturanthropologischer Perspektive. Frankfurt/Oder: Springer-Verlag.

HARGUINDEGUY, J. B. (2007). La frontière en Europe, un territoire?: coopération transfrontalière franco-espagnole. París: l'Harmattan.

Houtum, H. J. VAN (2000). «Introduction: current issues and debates on borders and border regions in European regional science». En: B. M. R. van der VelDE y H. VAN (eds.). Borders, regions and people. Londres: PION, 1-12.

KAPLAN, D. H. y HäKLI, J. (2002). Boundaries and place: European borderlands in geographical context. Lanham: Rowman \& Littlefield.

Letamendia, F. (coord.) (2011). Pueblos y fronteras en los Pirineos y el altiplano andino. Madrid: Fundamentos.

LONCÁN, A. H. (2002). «Infraestructuras y desarrollo económico en el Pirineo central (18502000)». Ager. Revista de Estudios sobre Despoblación y Desarrollo Rural, 2, 197-226.

MANCEBO, F. (1999). La Cerdagne et ses frontières : conflits et identités transfrontalières. Perpiñán: Llibres del Trabucaire.

Martinez, O. J. (2002). "The Dynamics of Border Interaction». En: Clive H. SCHOFIEL (ed.). Global boundaries: World boundaries. Vol. 1. Londres: Routledge, 1-15.

METIS (2018). EGTC Monitoring Report 2017. Bruselas: European Committee of the Regions. <https://doi.org/10.2863/506306>

Ministerio de Hacienda y de Administraciones Públicas (MHAP) (2014). La cooperación transfronteriza realizada por las entidades territoriales españolas. Madrid: 
Secretaría de Estado de Administraciones Públicas. <http://repositori.uji.es/xmlui/ bitstream/handle/10234/135945/Cooperaci\%C3\%B3n_transfronteriza_2014_04. pdf?sequence $=1$ \&isAllowed $=y>$ [Consulta: 6 de diciembre de 2016]

Moncusí, F. (2005). Fronteres, identitats nacionals i integració Europea. El cas de la Cerdanya. Valencia: Afers.

MoratA, F. (ed.). (2004). Gobernanza multinivel en la Unión Europea. Valencia: Tirant lo Blanch.

Observatori del Paisatge de Catalunya (2013). Le paysage transfrontalier de la Cerdagne. Un projet d'avenir commun. <http://www.catpaisatge.net/fitxers/tries/ proj_transfront_2013.pdf> [Consulta: 07 de noviembre de 2016].

O'DOWD, L. (2001). "The changing significance of European borders». Regional \& Federal Studies, 12 (4), 13-36. <https://doi.org/10.1080/714004774>

Oliveras, X. (2012). "La construcción de regiones transfronterizas: el caso de los mataderos de la Cerdanya (frontera franco-española) y Saarbrücken-Moselle Est (frontera franco-alemana)», XVI Coloquio de Geografía Rural (Sevilla, 10-12 de mayo de 2012).

- (2013). «La cooperación transfronteriza en la Cerdanya (frontera España-Francia)». Boletín de la Asociación de Geógrafos Españoles (AGE), 62, 25-48.

Oliveras, X.; DurÀ, A. y PERKMANN, M. (2010). «Las regiones transfronterizas: balance de la regionalización de la cooperación trasfronteriza en Europa (1958-2007)». Documents d'Anàlisi Geogràfica, 56 (1), 21-39.

PAASI, A. (1996). Territories, boundaries, and consciousness: The changing geographies of the Finnish-Russian boundary. Vol. 1. Chichester: Wiley.

- (1998). «Boundaries as social processes: Territoriality in the world of flows». Geopolitics, 3 (1), 69-88. <https://doi.org/10.1080/14650049808407608>

PaÜl, V.; Trillo-Santamaria, J. M. (2014). «Discussing the Couto Mixto (Galicia, Spain): transcending the territorial trap through borderscapes and border poetics analyses». Geopolitics, 20 (1), 56-78. <https://doi.org/10.1080/14650045.2013.857310>

PERKMANN, M. (2003). «Cross-border regions in Europe significance and drivers of regional cross-border co-operation». European Urban and Regional Studies, 10 (2), $153-171$. <https://doi.org/10.1177/0969776403010002004>

Rumley, D. y Minghi, J. V. (eds.). (1991). The geography of border landscapes. Londres: Routledge.

SAHLINS, P. (1989). Boundaries: the making of France and Spain in the Pyrenees. Oakland: University of California Press.

Sanabre, J. (1960). El Tractat dels Pirineus i la mutilació de Catalunya. Vol. 6. Barcelona: Barcino.

SANGUIN, A. L. (2013). «Euroregions and other eu's cross-border organizations: the risk of confusion, redundancy, oversizing and entropy. A critical assessment». Annales-Anali Za Istrske In Mediteranske Studije-Series Historia Et Sociologia, 23 (1), 155-164.

SCHelde, B. van der y HoeKyeld, G. A. (1992). "The regional development of borderlands: An explorative study in the Franco Italian Alps». Cashiers de Géographie du Québec, 36 (99), 483-500. $<$ https://doi.org/10.7202/022296ar> 
SoHn, C. (2014). "Modelling cross-border integration: The role of borders as a resource». Geopolitics, 19 (3), 587-608. <https://doi.org/10.1080/14650045.2014.913029>

SousA, L. D. (2013). «Understanding European cross-border cooperation: a framework for analysis». Journal of European Integration, 35 (6), 669-687.

Tulla, A. F. (1977). «Les deux Cerdagnes. Exemple de transformations économiques asymétriques de part et d'autre de la frontière des Pyrénées». Revue geographique des Pyrénées et du Sud-Ouest, 48 (4), 409-424.

- (1997). "La Cerdanya: dues realitats en un únic espai». En: J. Domingo y Ll. MAllart (eds.). A l'entorn de la frontera. Barcelona: Oikos Tau, 85-104.

VILA, P. (1926). La Cerdanya. Barcelona: Empúries, 1984.

Wolmer, W. (2003). «Transboundary conservation: the politics of ecological integrity in the Great Limpopo Transfrontier Park». Journal of Southern African Studies, 29 (1), 261-278.

Wilson, T. M. y Donnan, H. (eds.) (2012). A companion to border studies. Vol. 19. Chichester: Wiley.

\section{Páginas web}

AECT Hospital de la Cerdanya. <http://www.hcerdanya.eu> [Consulta: 10 de mayo de 2017].

IDESCAT. <www.idescat.cat> [Consulta: 07 de noviembre de 2016].

INSEE. <www.insee.fr> [Consulta: 07 de noviembre de 2016].

CEMR. <www.twinning.org> [Consulta: 14 de septiembre de 2016]. 